\title{
Low-cost equipment for schedule generation: II. Time-dependent schedules
}

\author{
R. GLYNN OWENS and L. J. AYLESBURY \\ University of Oxford, Oxford OX3 $7 J X$, England
}

\begin{abstract}
Complementary metal oxide semiconductor (CMOS) logic provides an efficient and convenient technology for the generation of schedules of reinforcement. A single circuit provides a module capable of use as either a fixed-interval timer or one generating a differential reinforcement of low-rate schedule. A second circuit provides for a programmable variableinterval generator with 16 independent intervals. Both modules are fully compatible with previously described circuits for ratio schedules.
\end{abstract}

An earlier paper (Aylesbury \& Owens, 1977) described the use of complementary metal oxide semiconductor (CMOS) integrated circuity to generate ratio-dependent reinforcement schedules. Of equal, if not greater, importance in the behavioral laboratory are schedules which require satisfaction of a temporal criterion. These include the fixed-interval (FI) schedule, in which reinforcement becomes available (contingent on a response) at regular intervals; the variable-interval (VI) schedule, in which reinforcement is programmed as in the FI schedule but with the added feature that the interval concerned varies from reinforcement to reinforcement; and the differential reinforcement of low rate (DRL) schedule, in which responses are reinforced only if a certain period of time has elapsed since the previous response.

For the purposes of this paper, FI and DRL equipment are considered together and separate from VI. The former are easily combined in a single module, whereas the VI, by virtue of the programming requirements is considerably more complex.

\section{FI AND DRL SCHEDULES}

Figure 1 shows the logical structure of a circuit providing these schedules. As with all the circuits to be described, it involves, from the user's viewpoint, only an input, an output, and a single control to set the parameter of the circuit. A switch determines the mode of operation (FI and DRL).

For FI operation the input is connected to the output through the network formed by NAND gates $2 \mathrm{C}$ and $2 \mathrm{D}$. The second input to NAND gate $2 \mathrm{C}$ is connected to the $\overline{\mathrm{Q}}$ output of flip-flop $1 \mathrm{~A}$ via the gate $5 \mathrm{~A}$ acting as a buffer. In the untriggered state, the $\bar{Q}$ output is high, enabling NAND gate $2 \mathrm{C}$ and allowing an input pulse

This work was completed while the first author was in receipt of a grant from the Medical Research Council of Great Britain, to whom thanks are due. Reprints may be obtained from R. Glynn Owens, University Department of Psychiatry, Warneford Hospital, Oxford OX3 7JX, England. to appear at the output. Such an input pulse simultaneously switches flip-flop $1 \mathrm{~A}$ via inverter 4A. This sets $Q$ to the level of D (i.e., high) and correspondingly sets $\bar{Q}$ low, which disables NAND gate $2 \mathrm{C}$ and prevents further pulses from passing. The $Q$ output remains high, enabling the multivibrator formed by NAND gates $2 \mathrm{~A}, 2 \mathrm{~B}$, and associated components to oscillate. The output of the multivibrator is fed to the input of the binary counter IC $3 \mathrm{~A}$; this continues until the count reaches 64 , when output $Q 7$ goes high, resetting flipflop $1 \mathrm{~A}$ via the $120 \mathrm{~K}$ resistor and stopping the multivibrator. $\overline{\mathrm{Q}}$ goes high, NAND gate $2 \mathrm{C}$ is again enabled, and the next pulse restarts the sequence. $\bar{Q}$ going high also resets counter $3 \mathrm{~A}$ to zero.

The frequency of the multivibrator, and hence the time taken for the counter to reset the flip-flop, is determined by the $2 \mathrm{M}$ potentiometer. This provides a means of controlling the interval. The range available varies according to the individual components used: The circuit of Figure 1 typically provides a range of intervals from 1 to $130 \mathrm{sec}$; longer time periods may be obtained by inserting a further counter between the output Q7 of IC3A and the $120 \mathrm{~K}$ resistor, the resets of both counters being tied together.

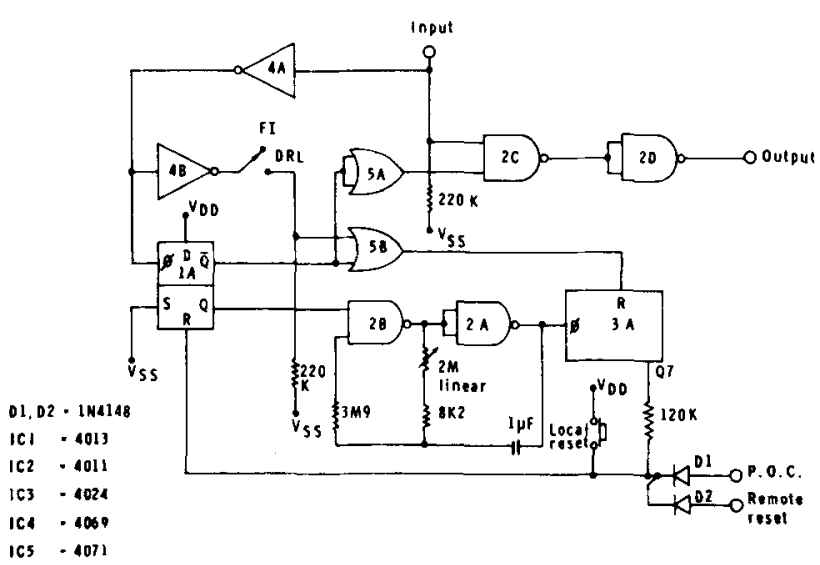

Figure 1. Logical structure of FI/DRL circuit. 


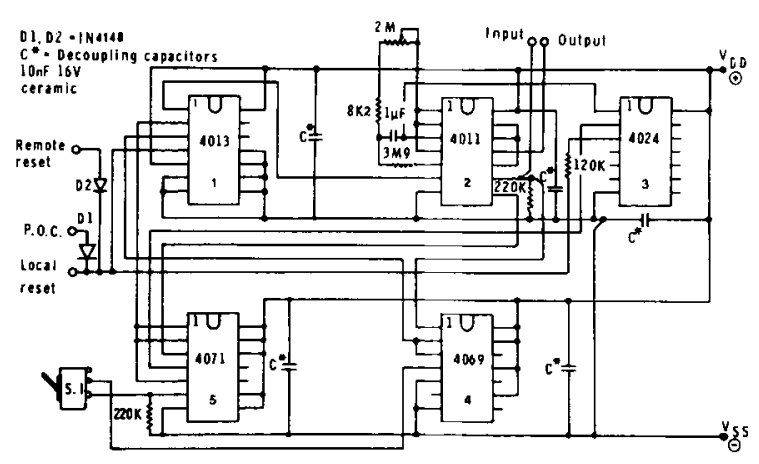

Figure 2. Wiring diagram for FI/DRL circuit.

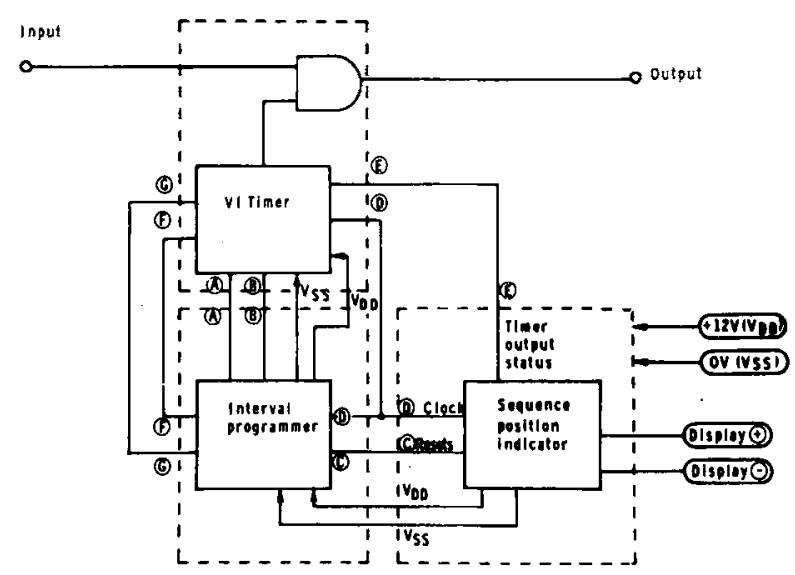

Figure 3. Overall structure of VI unit.

In the DRL mode the circuit works exactly as in FI, with the added proviso that any input resets the binary counter to zero, via inverters $4 A$ and $4 B$ and $O R$ gate 5B. Thus, an input before the time has elapsed resets the timing, requiring that no inputs occur during the period when the output circuit is disabled and providing DRL scheduling.

Figure 2 shows the circuit diagram for the module. The flip-flop is provided by the 4013, NAND gates by the 4011 , the counter by a 4024 , inverters by a 4069 , and the OR gates by a 4071 ;

\section{SCHEDULE}

The requirement of a programmable sequence in the VI module necessarily makes such a circuit more complex. The basic timing module works according to the same principles as the module used for the FI circuit. The difference is that the single potentiometer of the FI circuit is replaced by a series of potentiometers that are sequentially "switched in" to the circuit. In addition, it is convenient to have an indication of the point in the sequence that has been reached at any time during an experiment. Such information is provided by a position indicator unit that shows, with seven-segment displays, which of the series of potentiometers is currently switched in. Indication regarding the state of the output from the module is provided also, that is, whether or not the appropriate time interval has in fact expired.

Figure 3 shows the overall structure of the VI unit, with the interconnections between the three major subunits. As with the FI and DRL units, operation is simple, requiring only an input, an output, and the setting of the various potentiometers to give the times required. The interval programmer serves merely to switch in the various potentiometers, a process monitored by the position indicator module. The position indicator requires a more substantial power supply than the remaining circuits, due to the comparatively high current consumption of the seven-segment displays. Such a supply may be obtained from the auxillary output of the power supply previously reported (Aylesbury \& Owens, 1977).

Although the various units can be constructed on a single board, it is convenient to describe them as three separate units. The first of these, the VI timer, is merely a simplified version of the circuit used in the FI/DRL circuit: Its logical structure is shown in Figure 4. The mode of operation is as in the FI unit, with the following exceptions: (1) The potentiometer is removed, and

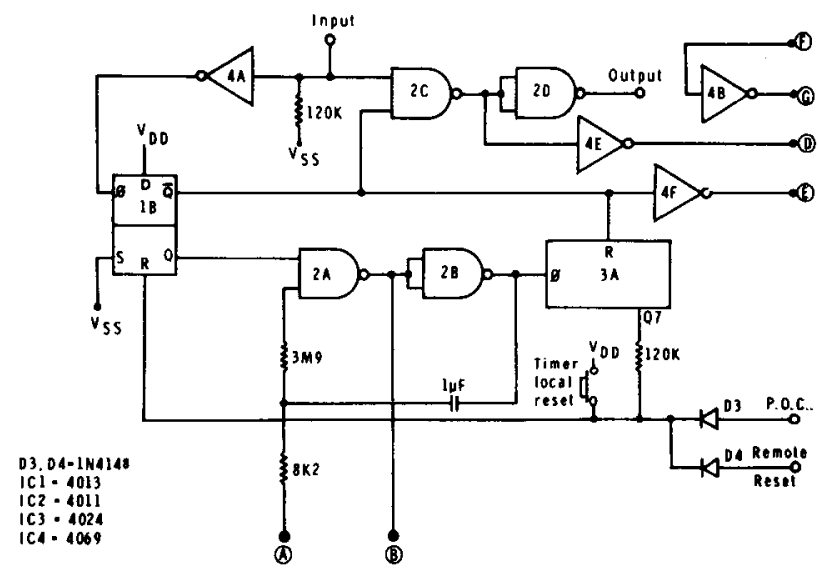

Figure 4. Logical structure of VI timer.

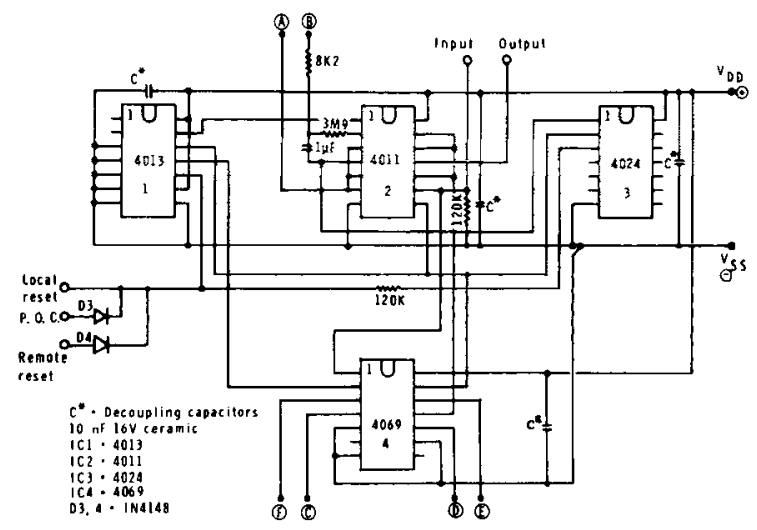

Figure 5. Wiring diagram for VI timer. 


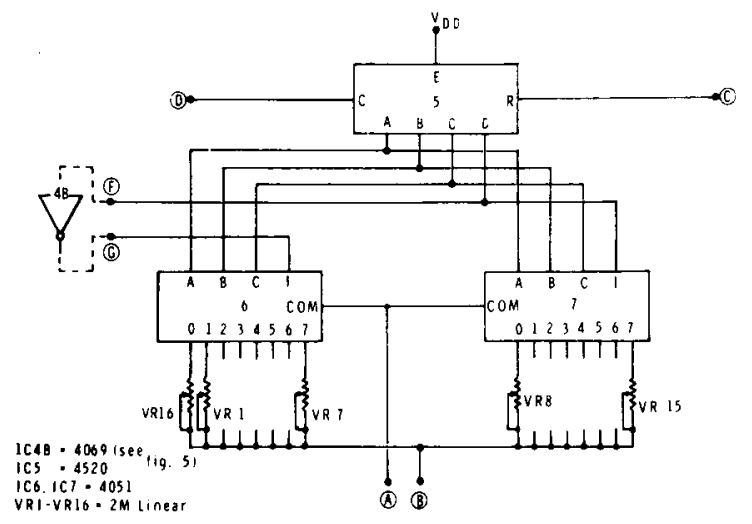

Figure 6. Logical structure of VI programming circuit.

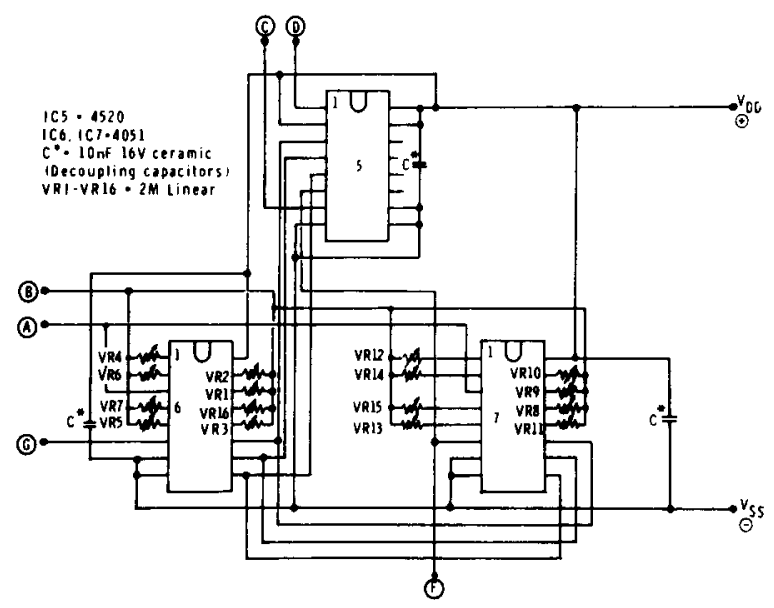

Figure 7. Wiring diagram of VI programmer.

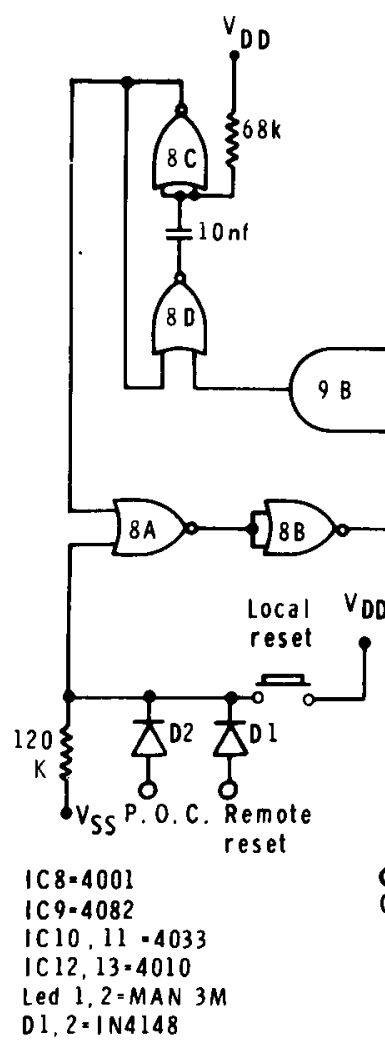

the points to which it would normally be connected are taken to the VI programming module; (2) the DRL option and its associated circuitry is removed; and (3) outputs are provided to the clock input of the position indicator (D) and the output status indicator of the position indicator module (E). Also shown in Figure 4 is the use of an inverter, otherwise unused in the 4069 package, in part of the programming circuit. The circuit diagram is shown in Figure 5.

To produce the programming circuit (Figure 6), it is convenient to use a type of integrated circuit, peculiar to the CMOS family, that contains "transmission gates." These may be considered as programmable switches functioning in such a way that no more than one of the eight outputs $0-7$ is connected to the common input (com) at any time: The particular output connected is that indicated by the binary number present at points A, B, and C. Input $I$ is an "inhibit" input, such that if a signal is present at $I$, all outputs $0-7$ are disconnected from the common.

The binary number at the input to ICs 6 and 7 is taken from the outputs of the binary counter IC5, the clock input of which is connected so as to increment at every output from the VI timer module, and the reset of which is taken from the position indicator module. Inverter IC4B is connected between the two inhibit inputs of IC6 and IC7 so that only one of them is able to function at any one time. The circuit sequentially switches on the potentiometers, each of which may be 


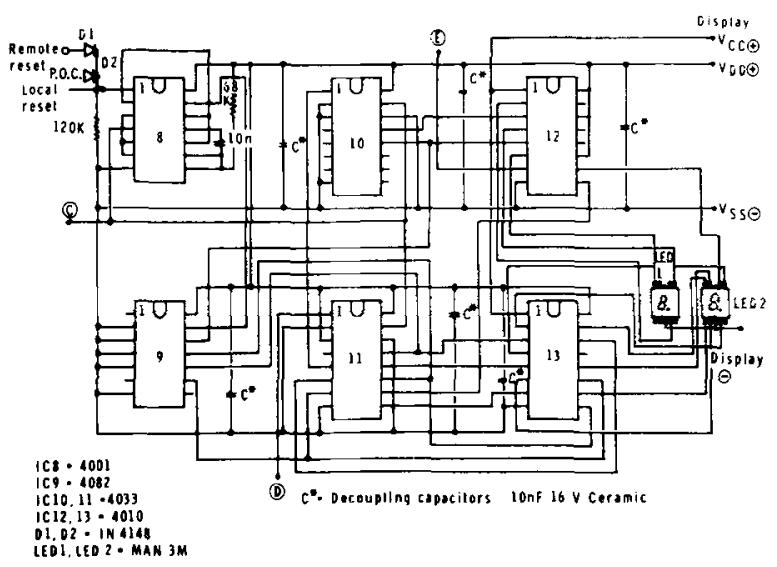

Figure 9. Wiring diagram for sequence position indicator.

independently calibrated and set to provide a 16-unit VI sequence. Figure 7 shows the circuit wiring diagram.

In order to detect which of the potentiometers is switched in at any time, the circuit illustrated in Figure 8 was devised. These two seven-segment displays are connected to the outputs of decade counters IC 10 and IC11, the former showing, by means of segment $f$, whether or not the time set on the potentiometer has expired (achieved by detecting and inverting via IC4F the state of $\bar{Q}$ of flip-flop 1 in Figure 4). The other segments of the displays are arranged to count from 0 to 15 , the clock input of the decade counters being connected, like the clock input of the binary counter IC5 (Figure 6), to the output of inverter 4E (Figure 4). Resetting of the sequence is obtained by sampling the outputs of the decade counters, segment b of IC10, and segments $\mathrm{c}$, $\mathrm{e}$, and $\mathrm{g}$ of IC11. Providing the inputs to the four-input AND gate IC9B, these can all be high only when the number 16 is present at the output. This triggers the monostable formed by NOR gate $8 \mathrm{C}, 8 \mathrm{D}$, and associated components, providing an input to NOR gate $8 \mathrm{~A}$, which with $8 \mathrm{~B}$ serves to provide resets to the various counters. The other input of NOR gate $9 \mathrm{~A}$ is provided by the remaining reset functions. The sevensegment display and drivers IC 12 and IC13 require a separate power supply. Connections are shown in Figures 8 and 9 , the associated wiring diagram for the position indicator circuit.

Ferster and Skinner (1957) point out that there are two ways in which such interval schedules can be programmed; a particular interval can start from the expiry of the previous interval or from the previous reinforcement. The circuits described here provide only the latter form of scheduling. To produce the former involves substantial complication of the circuitry. In practice, however, this should create little difficulty. Intervals timed from the expiry of the preceding interval, rather than from the preceding reinforcement, are rarely used. Moreover, interval schedules typically generate patterns of responding that make the functional effect of the two methods indistinguishable, with reinforcement generally being received immediately.

\section{REFERENCES}

Aylesbury, L. J., \& Owens, R. G. Low-cost equipment for schedule generation. I: Fixed and random ratio schedules. Behavior Research Methods \& Instrumentation, 1977, 9, 247-252.

Ferster, C. B., \& Skinner, B. F. Schedules of reinforcement. New York: Appleton-Century-Crofts, 1957.

(Received for publication August 25, 1977; revision accepted October 10, 1977.) 